\title{
LA INSPECCIÓN PRE-EMBARQUE COMO SISTEMA DE CONTROL A LA IMPORTACIÓN DE INSUMOS PROVENIENTES DE CHINA, DEL SECTOR METALMECÁNICO EN LIMA 2010-2015
}

\author{
PRE-SHIPMENT INSPECTION AS A CONTROL SYSTEM FOR THE IMPORTATION OF INPUTS \\ FROM CHINA, FROM THE METAL-MECHANICAL SECTOR IN LIMA 2010-2015
}

Yesenia Aguilar Lugo

Universidad Tecnológica del Perú

Licenciada en Administración de Negocios Internacionales de la Universidad Tecnológica del Perú, Lima, Perú

Correo electrónico: yeseniaal5@hotmail.com

[Recibido: 4/07/2018 Aceptado: 10/08/2018]

\section{RESUMEN}

Objetivo: Determinar en qué medida beneficia el sistema de control de inspección pre-embarque, aplicables en la gestión de importación de insumos provenientes de China para empresas del sector metalmecánico en Lima 2010-2015. Método: Tipo descriptivo, correlacional, No experimental transversal; y los sujetos u objetos de estudio, es el conjunto de empresas del sector metalmecánico de Lima Metropolitana, las conclusiones de esta investigación serán válidas para las empresas del sector metalmecánico de Perú. Resultados: De la investigación realizada al sector metalmecánica de Lima, se ha probado la hipótesis general con sus correspondientes hipótesis específicas, es decir que el sistema de control de inspección pre embarque beneficia significativamente a la gestión de importación de insumos provenientes de China para empresas del sector metalmecánico en Lima 2010-2015. Conclusiones: De los 18 encuestados de las empresas del rubro investigado se ha obtenido: el $94 \%$ de los encuestados considera que el sistema de control de inspección pre embarque beneficia significativamente a la gestión de importación de insumos provenientes de China para empresas del sector metalmecánico en Lima.

Palabras clave: Sistema, inspección, pre-embarque, importación, metalmecánica.

\begin{abstract}
Objective: To determine the extent to which the pre-shipment inspection control system benefits the management of imported inputs from China for companies in the metal-mechanic sector in Lima 2010-2015. Method: Descriptive, correlational, and of non-experimental - transverse type. The subjects or objects of study, are the set of companies in the metal-mechanic sector of Metropolitan Lima. The conclusions of this investigation will be valid for companies in the metal-mechanic sector of Peru. Results: The research was carried out in the metal-mechanic sector of Lima. The general hypothesis with its corresponding specific hypotheses has been tested, that is to say that the pre-shipment inspection control system significantly benefits the import management of inputs from China for companies of the metal-mechanic sector in Lima 2010-2015. Conclusions: Of the 18 companies that have been researched $94 \%$ of respondents believe that the pre-shipment inspection control system significantly benefits the import management of inputs from China for companies in the metal-mechanic sector of Lima.
\end{abstract}

Keywords: System, inspection, pre-shipment, import, metalworking. 


\section{INTRODUCCIÓN}

El objetivo es determinar, en qué medida beneficia el sistema de control de inspección pre-embarque aplicables en la gestión de importación de insumos provenientes de China, para empresas del sector metalmecánico en Lima 2010-2015.

A continuación, se mostrará algunas definiciones relacionadas a las variables del sistema de control de Inspección pre-embarque e importación de insumos provenientes de China por empresas del sector metalmecánico.

En el comercio internacional se efectúa un intercambio entre bienes y servicios que se realizan a través de los distintos países. Lerma y Márquez (2010) definen:

El comercio internacional, comprende tanto a la exportación, que corresponde a la venta de los productos (bienes y servicios) originarios de un país a clientes situados en otro país, como la importación, que es la compra de bienes o servicios a un proveedor originario de un país distinto al del comprador. (pág. 7).

Para Krugman y Obstfeld (2006), “a principios del siglo XXI los países están mucho más estrechamente relacionados mediante el comercio de bienes y servicios, mediante los flujos de dinero, y mediante las inversiones en las economías de cada uno" (pág. 1).

Según Krugman y Obstfeld (2006), la teoría de la economía internacional se divide en dos grandes campos:

El estudio del comercio internacional y el estudio de las finanzas internacionales. El análisis del comercio internacional pone el acento en las transacciones reales de la economía internacional, es decir, en aquellas transacciones que implican un movimiento físico de bienes o un compromiso tangible de recursos económicos. El análisis financiero internacional pone el acento en el lado monetario de la economía internacional, esto es, en las transacciones financieras como las compras por parte de los extranjeros de dólares estadounidenses. (pág. 8).

Por otro lado, para Amaya (2017), el comercio internacional es el movimiento de intercambio comercial, monetario y entre otras operaciones que intervienen en el flujo comercial de diferentes naciones, cabe precisar que aquí también participan las exportaciones e importaciones que realiza un país con los diferentes bloques eco- nómicos como por ejemplo entre Perú y la Comunidad Andina, Mercado Común del Sur, entre otros.

La inspección pre-embarque o inspección antes de la expedición, se define como la "práctica que consiste en emplear a empresas privadas para verificar pormenores de la expedición como el precio, la cantidad y la calidad de las mercancías pedidas al extranjero". (Organzación Mundial del Comercio, 2017). Cuando las empresas del sector metalmecánico realizan una importación de insumos desde el país de China, y emplean el sistema de inspección pre-embarque, como herramienta de gestión de importación en el comercio internacional, dicho sistema debe cumplir estándares de calidad que garanticen el cumplimiento de los requerimientos solicitados en el contrato de compra y venta internacional, para lo cual el sistema de inspección pre-embarque utiliza la norma AQL o Nivel Aceptable de Calidad, entendemos sobre esta norma de calidad.

El Instituto Nacional Estadounidense de Estándares (ANSI) / Sociedad Americana para la Calidad (ASQ) Z1.4-2008: Procedimientos de muestreo y tablas para la inspección por atributos. Proporciona instrucciones simples sobre cómo seleccionar correctamente el plan de muestreo en función del tamaño de la población y el riesgo aceptable. Además, este documento proporciona una descripción general de las estadísticas detrás del desarrollo de planes de muestreo. (Eugenie Webster, 2013).

Es muy importante tener en cuenta la definición del régimen aduanero Importación para el consumo, según SUNAT:

De acuerdo al Art. $49^{\circ}$ de la Ley General de Aduanas aprobada mediante el D.L. No 1053; La Importación para Consumo, es el régimen aduanero que aprueba el ingreso de mercancías al territorio aduanero para su consumo, después de haberse cumplido con el pago o garantía respectivo, además de los derechos arancelarios e impuestos aplicables, de acuerdo al cumplimiento de las formalidades y otras obligaciones aduaneras. (SUNAT, 2015).

Ante esto es imprescindible la necesidad de conocer el proceso de destinación aduanera, el Artículo $130^{\circ}$ de la ley general de aduanas, aprobada mediante el Decreto Legislativo No 1235. Define:

La destinación aduanera es solicitada por medio de- 
claración aduanera por los despachadores de aduana o demás personas con su respectiva autorización legal. Las declaraciones se tramitan bajo las siguientes modalidades de despacho aduanero y plazos.

a. Anticipado: se contabiliza treinta días (30) antes de la llegada del medio de transporte;

b. Diferido: al término de la descarga al día siguiente, se genera en un plazo calendario de quince (15) días;

c. Urgente: Dentro del plazo de quince (15) días calendario antes de la llegada del medio de transporte y hasta siete (07) días calendario posteriores a la fecha del término de la descarga. (SUNAT, 2015)

De acuerdo con SUNAT (2010), respecto con el inciso a), la mercancía debe arribar teniendo como plazo no menor o igual a treinta (30) días calendario, contabilizados a partir de la fecha del día siguiente en que se numera la declaración aduanera; al finalizar el plazo la mercancía se someterá al despac ho diferido, salvo caso fortuito deben ser acreditados ante la Administración Aduanera, de acuerdo al reglamento. Para el inciso b) la mercancía en caso caiga en abandono legal, estas podrán ser sometidos a los regímenes aduaneros según Reglamento de este.

Para la presente investigación, se analizó el sector metalmecánico de Lima, porque concentra gran parte de las empresas de este sector, cabe recalcar que existen comités de metalmecánico en los diferentes gremios empresariales; La Sociedad Nacional de Industrias (2014) define a los Comités Metalmecánicos, como un gremio industrial fundado en el año 1958, para en cierta manera respaldar y cuidar los intereses de la pequeña, mediana y gran industria metalmecánica, a su vez brindar apoyo para su crecimiento en conjunto con la mejora de posicionamiento de la economía peruana.

El desarrollo de la investigación se justifica en los siguientes aspectos fundamentales:

La limitación más importante de este trabajo es la escasez de información sobre el sistema de inspección pre-embarque en la gestión de importación de insumos provenientes de China para empresas del sector metalmecánico, por lo que se hace necesario abordar este tema ya que el estudio puede servir de fuente a trabajos similares.

Este país (China), se presenta ante muchos empresarios y emprendedores como la solución a sus requeri- mientos de insumos a costos muy asequibles. Según Xalca Perú, indica que entre el 2010 y el 2016:

Las importaciones provenientes de China han crecido a una tasa promedio anual de $8,2 \%$, alcanzando en el 2016 un valor de US\$ 8.239 millones, de los cuales el 99,6\% corresponde a productos no tradicionales. Los principales productos que se importan de China son metalmecánicos (51,4\%), químicos (12,7\%), textiles y confecciones $(10,9 \%)$ y siderometalúrgicos $(8,8 \%)$. (Xalca Perú, 2017).

Los empresarios del sector metalmecánico realizan la importación por el costo reducido de los insumos, sin embargo, el estudio de investigación muestra las falencias del producto chino, a la hora de valorar su rentabilidad de operación. Entre las más representativas esta los costos y su complejidad en el proceso, a la hora que se tiene que realizar la importación de China, tales como: Estándares de calidad inadecuados, cuando el empresario realiza el proceso de importación al Perú y el producto no cumple, con las especificaciones técnicas o con las características detalladas en la compra, iniciándose el proceso de informalidad en la gestión de importación de insumos provenientes de China. Según Cristóbal (2014), el sistema de inspección pre-embarque es beneficioso, "Esta inspección ofrece a los clientes la certeza de que las mercancías que llegan a su lugar de destino conformes con las especificaciones requeridas. Tales especificaciones pueden provenir, tanto de los propios de los clientes como requisitos legales." (pág. 112).

La inspección pre-embarque, disminuye la informalidad y contribuye a dar seguridad al proceso de compra internacional. Según Cristóbal (2014):

Las inspecciones de mercancías, cualquiera que sea la modalidad, son capaces de cubrir una de las necesidades más importantes de los operadores del comercio exterior: La seguridad de lo que se ha importado/exportado coincide plenamente con el objeto de la transacción comercial internacional. (pág. 109).

El análisis de este tema de investigación genera un aporte en el campo del Comercio Exterior, nos dará un panorama del proceso de la aplicación de un sistema de control, en el proceso de gestión de importación de insumos provenientes de China al Perú, en el sector metalmecánico. 


\section{MATERIAL Y MÉTODOS}

En la investigación, el método de estudio ha sido enmarcado dentro del enfoque descriptivo y correlacional; primero se identificaron las variables elegidas para la investigación cumpliendo la parte descriptiva y luego se establece una relación entre variables del estudio sin poder establecer un sentido de causalidad o relaciones causales, fundamentados en el planteamiento.

La investigación es no experimental y transversal, no experimental porque no se manipulará las variables de la investigación, limitándose el investigador a una observación estructurada. Y transversal, debido a que la observación y el recojo de datos se realizarán en un momento único, debido a las limitaciones temporales y presupuestarias para la presente investigación.

La población de estudio, se considera el tamaño del universo y es asequible al alcance de la investigación, nos permite hacer por conveniencia, es decir, considerar a todo el universo como muestra, a las 18 empresas del sector metalmecánico ubicadas en el departamento de Lima, afiliados al gremio representativo de los exportadores y que realizan importación de insumos provenientes del país de China. Esto es debido también a que, aplicando las respectivas fórmulas para determinar el tamaño muestral, este casi coincide con el tamaño de la población, por lo que se pierde la ventaja de buscar una muestra. La mues- tra serán las empresas del sector metalmecánico de Lima, se ha definido a conveniencia, y ha estado conformada por: Gerentes generales, jefes de logísticas y personal que labora en el área de comercio exterior.

Para la recolección de datos, en la presente investigación se emplea la técnica de la encuesta, usando como instrumento el cuestionario. Cabe precisar que, para el análisis de los datos cuantitativos obtenidos, utilizaremos como herramienta la estadística descriptiva, a través de software estadístico. Finalmente, la data cuantitativa obtenida se presenta en tablas y gráficos, interpretando los resultados relevantes y de interés.

\section{RESULTADOS}

Respecto a la Hipótesis General: El sistema de control de inspección pre embarque beneficia significativamente a la gestión de importación de insumos provenientes de China para empresas del sector metalmecánico en Lima 2010-2015, se dirigió la siguiente pregunta: ¿Dichas medidas (Sistema de Inspección pre Embarque) comprobables de los resultados permitirán determinar la eficacia de una adecuada gestión de importación?, se determinó que el $94 \%$ de los encuestados considera que el sistema de inspección pre embarque beneficia significativamente la eficacia de una adecuada gestión de importación. Tal como se observa en:

Tabla 1.

Dichas medidas (Sistema de Inspección pre embarque) comprobables de los resultados permitirán determinar la eficacia de una adecuada gestión de importación.

\begin{tabular}{ccccc}
\hline & Respuesta & Frecuencia & Porcentaje & $\begin{array}{c}\text { Porcentaje } \\
\text { válido }\end{array}$ \\
\hline \multirow{3}{*}{ Validos } & SI & 17 & $94 \%$ & $94 \%$ \\
& NO & 1 & $6 \%$ & $6 \%$ \\
& Total & 18 & $100 \%$ & $100 \%$ \\
\hline
\end{tabular}

Fuente: Cuadro excel / Elaboración propia.

\section{Hipótesis Específica 1}

La implementación de control de inspección pre-embarque de la importación de insumos proveniente de Chi- na a Lima Metropolitana permitirá a las empresas del sector metalmecánico reducir significativamente sus costos y aumentar sus ganancias, se dirigió la siguiente pregunta: ¿Dichas medidas contribuyen a reducir de costos y a opti- 
mizar los beneficios de las empresas importadoras de insumos por empresas del sector metalmecánico de Lima? Y se llegó a determinar que el 83\% de las 18 empresas encuestadas, considera la existencia de una relación positiva respecto a reducir costos y a optimizar los beneficios de las empresas del sector metalmecánico de Lima, según se muestra:

\section{Tabla 2.}

Dichas medidas contribuyen a reducir costos y a optimizar beneficios de las empresas importadoras de insumos por empresas del sector metalmecánico de Lima.

\begin{tabular}{rrccc}
\hline & Respuesta & Frecuencia & Porcentaje & $\begin{array}{c}\text { Porcentaje } \\
\text { válido }\end{array}$ \\
\hline \multirow{3}{*}{ Validos } & SI & 15 & $83 \%$ & $83 \%$ \\
& NO & 3 & $17 \%$ & $17 \%$ \\
& Total & & $100 \%$ & $100 \%$ \\
\hline
\end{tabular}

Fuente: Cuadro excel / Elaboración propia.

\section{Hipótesis Específica 2}

El grado de cumplimiento de los requisitos técnicos mediante la inspección pre embarque influye significativamente la conformidad de los clientes en cuanto a las órdenes de compra en la importación de insumos proveniente de China a Lima Metropolitana por empresas del sector metalmecánico, se dirigió la siguiente pregunta: ¿El sistema de control de inspección pre embarque de insumos por empresas del sector metalmecánico de Lima permitirá mejorar el cumplimiento de las especificaciones técnicas?, el $94 \%$ de los encuestados considera que el sistema de control de inspección pre embarque de insumos por empresas del sector metalmecánico de Lima permitirá mejorar el cumplimiento de las especificaciones técnicas, esto indica que la mayoría de las empresas califica como positiva el sistema de inspección pre embarque dado que mejora el cumplimiento de las especificaciones técnicas. Según se muestra:

Tabla 3.

El sistema de control de inspección pre embarque de insumos por empresas del sector metalmecánico de Lima permitirá mejorar el cumplimiento de las especificaciones técnicas.

\begin{tabular}{ccccc}
\hline & Respuesta & Frecuencia & Porcentaje & $\begin{array}{c}\text { Porcentaje } \\
\text { válido }\end{array}$ \\
\hline \multirow{3}{*}{ Validos } & SI & 17 & $94 \%$ & $94 \%$ \\
& NO & 1 & $6 \%$ & $6 \%$ \\
& Total & & $100 \%$ & $100 \%$ \\
\hline
\end{tabular}

Fuente: Cuadro excel / Elaboración propia.

\section{Hipótesis Específica 3}

El sistema de inspección pre-embarque no se convierte en un sobrecosto para la importación de insumos provenientes de China para empresas del sector metalmecánico en Lima, se dirigió la siguiente pregunta: ¿ ¿Dichas medidas contribuyen a la optimización de costos en la importación de insumos por empresas del sector metalmecánico de Lima?, determinaron que $61 \%$ de los encuestados, está de acuerdo que dichas medidas contribuyen a la optimización de costos en la importación de insumos por empresas del sector metalmecánico de Lima. Según: 
Tabla 4.

Dichas medidas contribuyen a la optimización de costos en la importación de insumos por empresas del sector metalmecánico de Lima.

\begin{tabular}{crccc}
\hline & Respuesta & Frecuencia & Porcentaje & $\begin{array}{c}\text { Porcentaje } \\
\text { válido }\end{array}$ \\
\hline \multirow{3}{*}{ Validos } & SI & 11 & $61 \%$ & $61 \%$ \\
& NO & 7 & $39 \%$ & $39 \%$ \\
& Total & & $100 \%$ & $100 \%$ \\
\hline
\end{tabular}

Fuente: Cuadro excel / Elaboración propia.

En la Figura 1 se muestra la comprobación de la hipótesis general con sus respectivas hipótesis específicas de la investigación realizada, se observa que el $94 \%$ de los encuestados considera que el sistema de control de inspección pre-embarque beneficia considerablemente en la importación de insumos provenientes de China, mientras que el $83 \%$ indica que dicho sistema reducirá costos y optimizará los beneficios de las empresas, además un $94 \%$ precisa que el sistema de inspección pre embarque afecta de manera favorable en el cumplimiento de las especificaciones técnicas y finalmente el $61 \%$ de los encuestados considera que este sistema de inspección favorece a la optimización de costos en el proceso de la importación de insumos por empresas del sector metalmecánico de Lima.

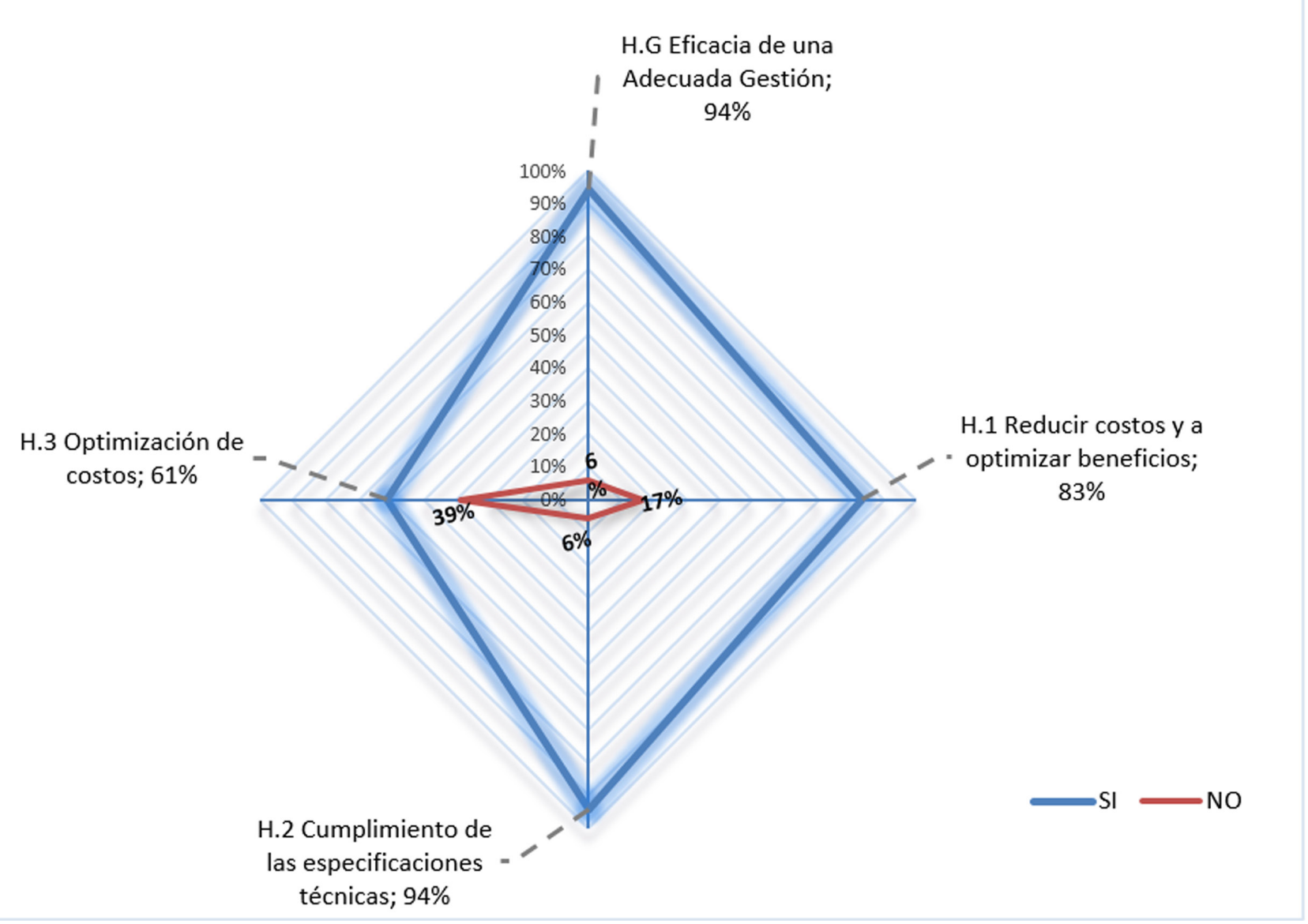

Figura 1. Comprobación de la hipótesis general con las respectivas hipótesis específicas. Fuente: Excel / Elaboración propia. 


\section{DISCUSIÓN}

Del análisis realizado y resultados obtenidos el sistema de control de inspección pre embarque beneficia significativamente a la gestión de importación de insumos provenientes de China para empresas del sector metalmecánico en Lima. Siendo una gran ventaja para el importador peruano conocer los beneficios de utilizar un sistema de inspección pre embarque que le permita mejorar su gestión de importación, cabe precisar que el grado de cumplimiento de los requisitos técnicos mediante la inspección pre embarque influye significativamente la conformidad de los clientes en cuanto a las órdenes de compra en la importación de insumos proveniente de China a Lima Metropolitana por empresas del sector metalmecánico ya que la conformidad de los insumos importados garantiza al importador que los requisitos solicitados cumplan las especificaciones de las normas y regulaciones técnicas establecidas.

Además, la implementación de un sistema de control de inspección pre embarque de la importación de insumos proveniente de China a Lima Metropolitana permitirá a las empresas del sector metalmecánico reducir significativamente sus costos y aumentar sus ganancias. Dicho sistema de control de inspección pre embarque permite que las empresas sean competitivas al importar de China país donde las fábricas son capaces de crear una gran variedad de insumos de calidad, usualmente con precios más bajos que cualquier otro lugar.

El sistema de inspección pre-embarque no se convierte en un sobrecosto para la importación de insumos provenientes de China para empresas del sector metalmecánico en Lima. Dicho sistema ayuda a que las empresas logren la satisfacción de sus clientes con el menor costo posible ya que están importando insumos a precios competitivos.

\section{REFERENCIAS BIBLIOGRÁFICAS}

Amaya F., A. D. (2017). Aldo Amaya (2017). Comercio Internacional, Teoría, Casos y Operatividad del Comercio. (Vol. Primera Edición). Lima, Perú: Grupo Universitario S.A.C.

Cristobal Carle, G. (2014). Internacionalización, mercados y empresa. Madrid, España: ESIC EDITORIAL.
Eugenie Webster. (24 de Noviembre de 2013). IVT NETWORK. Obtenido de http://www.ivtnetwork.com/article/sampling-attributes-using-ansiasq-z14-2008-standard

Industrias, S. N. (30 de Agosto de 2014). Sociedad Nacional de Industrias. Obtenido de http://www.cmm.org.pe/

Jerez, J. L. (2007). El comercio Internacional (Vol. $3^{\text {a }}$ ). Madrid, España: ESJC Editorial.

KRUGMAN, P. R., \& OBSTFELD, M. (2006). ECONOMÍA INTERNACIONAL Teoría y política (Vol. SÉPTIMA EDICIÓN). (A. Cañizal, Ed., \& Y. Moreno, Trad.) Madrid: PEARSON EDUCACIÓN, S.A. Obtenido de http://webdelprofesor.ula.ve/economia/oscared/materias/E_E_Mundial/Economia_Internacional_Krugman_Obstfeld.pdf

Lerma Kirchner, A. E., \& Márquez Castro, E. (2010). Comercio y marketing internacional (Vol. Cuarta Edición). Ciudad de Mexico: Cengage Learning Editores, S. A. de C. V.

Organzación Mundial del Comercio. (03 de 04 de 2017). OMC. Obtenido de https://www.wto.org/spanish/tratop_s/preship_s/preship_s.htm

SUNAT. (01 de Octubre de 2010). www.sunat.gob.pe. Obtenido de http://www.sunat.gob.pe/legislacion/proce$\mathrm{dim} /$ normasadua/gja-03.htm

SUNAT. (26 de Setiembre de 2015). www.sunat.gob.pe. Obtenido de http://www.sunat.gob.pe/legislacion/procedim/normasadua/gja-03.htm

SUNAT. (06 de Diciembre de 2015). www.sunat.gob.pe. Obtenido de http://www.sunat.gob.pe/legislacion/procedim/despacho/importacion/importacA/procGene$\mathrm{ral} /$

Xalca Perú. (30 de Abril de 2017). La República. El TLC entre Perú y China, siete años después de su firma, pág. s/p. Obtenido de https://larepublica.pe/economia/870705-el-tlc-entre-peru-y-china-siete-anos-despues-de-su-firma 
